City University of New York (CUNY) CUNY Academic Works

\title{
Literature and Psychoanalysis
}

Elisabeth S. von Uhl

CUNY City College

\section{How does access to this work benefit you? Let us know!}

More information about this work at: https://academicworks.cuny.edu/cc_oers/343

Discover additional works at: https://academicworks.cuny.edu

This work is made publicly available by the City University of New York (CUNY).

Contact: AcademicWorks@cuny.edu 


\section{FIQWS 10108 Literature and Psychoanalysis, Writing Section, Fall 2020}

HA7: $11 \mathrm{am}-12: 15 \mathrm{pm}$

HA8: $12: 30 \mathrm{pm}-1: 45 \mathrm{pm}$

evonuhl@ccny.cuny.edu

https://literatureandpsychoanalysis.commons.gc.cuny.edu

Weekly announcements will be posted every Monday via BB.

The schedule is subject to change and please let me know if links do not work.

\begin{tabular}{|c|c|c|c|}
\hline $\begin{array}{l}\text { Week } \\
\text { dates: }\end{array}$ & Read/Watch: & Assignments: & Due Dates: \\
\hline $\begin{array}{l}\text { Wednesday - } \\
8 / 26 \\
\text { Wed } 8 / 26 \\
\text { Synchronous } \\
\text { Zoom class }\end{array}$ & $\begin{array}{l}\text { Review: Syllabus and CUNY } \\
\text { Commons page } \\
\text { Quick Start for Blackboard } \\
\text { Learn (and bookmark!) } \\
\text { CUNY Academic Commons } \\
\text { Help (and bookmark!) } \\
\text { E-mail Guidelines for } \\
\text { Students } \\
\text { Online Forums: Responding } \\
\text { Thoughtfully }\end{array}$ & $\begin{array}{l}\text { - } \\
\text { - In-take Journal Entry found } \\
\text { on Professor Yankwitt's BB (I } \\
\text { will have access to this } \\
\text { information and it will help } \\
\text { me be a better teacher, so } \\
\text { please be honest.) }\end{array}$ & $\begin{array}{l}\text { DUE } 8 / 30 \\
\text { by } 11: 59 \mathrm{pm}\end{array}$ \\
\hline Mon. 8/31 ... & $\begin{array}{l}\text { Rhetorical Context* } \\
\text { Rhetorical Terms and their } \\
\text { definitions: } \\
-\quad \begin{array}{l}\text { Stance and } \\
\text { Language }\end{array} \\
-\quad \text { Tone and Purpose } \\
-\quad \text { Medium and Design } \\
\text { Discussion of Genre: } \\
\text { Navigating Genres } \\
\text { "Troubleshoot Your } \\
\text { Reading": }\end{array}$ & $\begin{array}{l}\text { - Extra Credit Discussion } \\
\text { Board Post } \\
\text { - Group worksheet }\end{array}$ & $\begin{array}{l}\text { Due } 9 / 6 \text { by } \\
11: 59 \mathrm{pm}\end{array}$ \\
\hline
\end{tabular}

This work is licensed under the Creative Commons Attribution-NonCommercial 4.0 International License. To view a copy of this license, visit http://creativecommons.org/licenses/by-nc/4.0/ or send a letter to Creative Commons, PO Box 1866, Mountain View, CA 94042, USA. 


\begin{tabular}{|c|c|c|c|}
\hline & $\begin{array}{l}\text { Troubleshoot Your Reading } \\
\text { - The Word on College } \\
\text { Reading and Writing } \\
\text { Watch: } \\
\text { Understanding Assignments } \\
\text { - UNC Writing Center }\end{array}$ & & \\
\hline $\begin{array}{l}\text { Mon. 9/7... } \\
\text { (No class on } \\
\text { Monday, 9/7) } \\
\text { Wed. 9/9: } \\
\text { Synchronistic } \\
\text { Zoom Class }\end{array}$ & 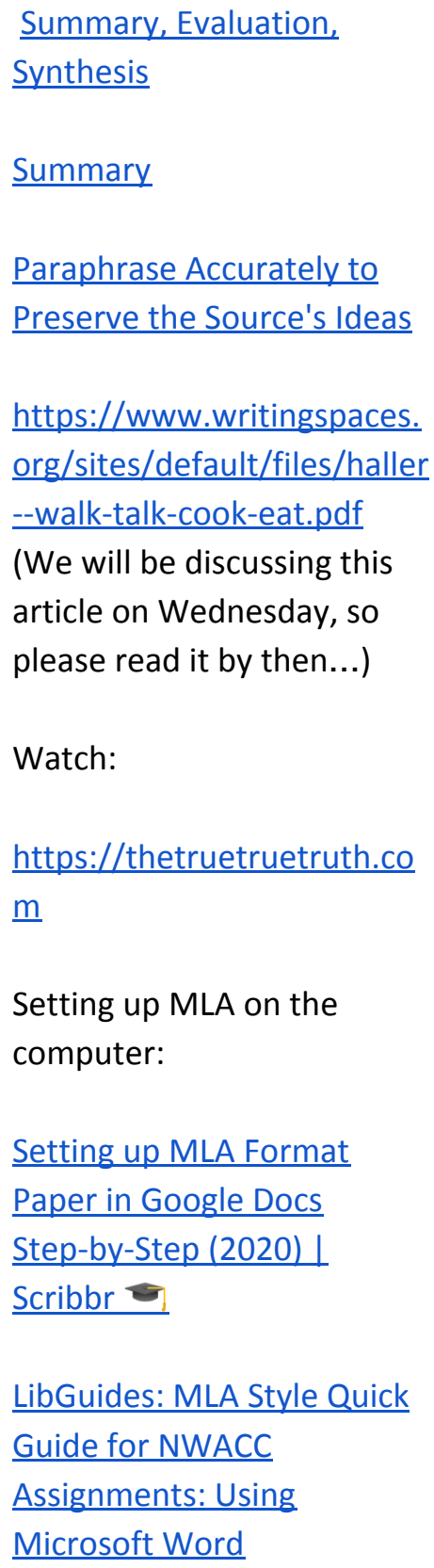 & $\begin{array}{l}\text { - } \quad \text { Blog Post } \\
\text { - } \text { Peer Review } \\
\text { - } \text { Discussion Board } \\
\text { Sign up for CUNY Commons } \\
\text { website and adjust your } \\
\text { privacy settings }\end{array}$ & $\begin{array}{l}\text { Due: Sun. } \\
9 / 13\end{array}$ \\
\hline
\end{tabular}

This work is licensed under the Creative Commons Attribution-NonCommercial 4.0 International License. To view a copy of this license, visit http://creativecommons.org/licenses/by-nc/4.0/ or send a letter to Creative Commons, PO Box 1866, Mountain View, CA 94042, USA. 


\begin{tabular}{|c|c|c|c|}
\hline Mon. 9/14 & $\begin{array}{l}\underline{\text { Peer Review }} \\
\frac{\text { BAD IDEAS }}{\text { (pg. 253-257) }} \\
\text { Watch: } \\
\underline{\text { IWE: Genre Analysis and }} \\
\underline{\text { Reverse Outlining }} \\
\underline{\text { IWE: Sentences }} \\
\underline{\text { How We Cite - UNC Writing }} \\
\underline{\text { Center }}\end{array}$ & $\begin{array}{ll}\circ & \text { Peer Review } \\
\circ & \text { Discussion Board } \\
\circ & \text { MLA Quiz } \\
& \text { (assignment post on } \\
\text { BB) }\end{array}$ & $\begin{array}{l}\text { Due: Sun. } \\
9 / 20 \\
\text { Summary and } \\
\text { Response } \\
\text { Due }\end{array}$ \\
\hline $\begin{array}{l}\text { Mon. 9/21 } \\
\text { Wed. 9/23 } \\
\text { Synchronistic } \\
\text { Zoom Class }\end{array}$ & $\begin{array}{l}\text { Read: } \\
\text { Bitzer's "The Rhetorical } \\
\text { Situation" (PDF on BB) } \\
\text { Research } \\
\text { Watch: } \\
\text { IWE: Punctuation }\end{array}$ & $\begin{array}{ll}\text { - } & \text { Blog Post } \\
\text { - } & \text { Peer Review } \\
\text { - } & \text { Discussion Board }\end{array}$ & \\
\hline $\begin{array}{l}\text { Mon. 9/28 } \\
\text { (Mon. 9/28 No } \\
\text { Class and } \\
\text { Tues 9/29 } \\
\text { Follows } \\
\text { Monday } \\
\text { schedule) }\end{array}$ & $\begin{array}{l}\text { Read: } \\
\text { Thesis, Research Question, } \\
\text { Hypothesis, Title } \\
\underline{\text { MLA Style }} \\
\underline{\text { Help...I've Been Asked to }} \\
\text { Synthesize! } \\
\text { Watch: }\end{array}$ & $\begin{array}{l}\text { - Peer Review } \\
\text { - Discussion Board }\end{array}$ & \\
\hline
\end{tabular}

This work is licensed under the Creative Commons Attribution-NonCommercial 4.0 International License. To view a copy of this license, visit http://creativecommons.org/licenses/by-nc/4.0/ or send a letter to Creative Commons, PO Box 1866, Mountain View, CA 94042, USA. 


\begin{tabular}{|c|c|c|c|}
\hline & $\begin{array}{l}\text { IWE: Paragraph Organization } \\
\text { and Flow } \\
\text { Listen: } \\
\underline{\text { Inside the mind of the }} \\
\underline{\text { bullshitter: Science Weekly }} \\
\text { podcast }\end{array}$ & & \\
\hline $\begin{array}{l}\text { Mon. 10/5 } \\
\text { Wed. 10/ } 7 \\
\text { Synchronistic } \\
\text { Zoom Class }\end{array}$ & $\begin{array}{l}\text { Read: } \\
\text { Rhetorical Analysis in the } \\
\text { Real World: A Useful } \\
\frac{\text { Thinking Tool }}{\text { (We will be discussing this }} \\
\text { reading on Wednesday, so } \\
\text { have it read by then...) } \\
\text { Grammar Essentials } \\
\text { Watch: } \\
\underline{\text { WWE: Ways to Approach }} \\
\text { Revision }\end{array}$ & $\begin{array}{ll}\text { - } & \text { Blog Post } \\
\text { - } & \text { Peer Review } \\
\text { - } & \text { Discussion Board }\end{array}$ & \\
\hline Mon. 10/12 & $\begin{array}{l}\text { Read: } \\
\text { Annoying Ways People Use } \\
\underline{\text { Sources }} \\
\text { Editing } \\
\text { Watch: Voice Markers } \\
\text { Library }\end{array}$ & $\begin{array}{l}\text { - Peer Review } \\
\text { - } \quad \text { Discussion Board }\end{array}$ & \\
\hline $\begin{array}{l}\text { Mon. 10/19 } \\
\text { Wed. 10/21 } \\
\text { Synchronistic } \\
\text { Zoom Class }\end{array}$ & $\begin{array}{l}\text { Read: } \\
\underline{\text { https://wac.colostate.edu/d }} \\
\text { ocs/books/writingspaces2/r } \\
\text { eid--ten-ways-to-thin } \\
\underline{\text { K.pdf }}\end{array}$ & $\begin{array}{ll}\text { - } & \text { Blog Post } \\
\text { - } & \text { Peer Review } \\
\text { - } & \text { Discussion Board } \\
\text { - } & \text { Sign up for individual } \\
& \text { meeting }\end{array}$ & $\begin{array}{l}\text { Due: Sun } \\
10 / 25 \\
\text { Exploratory } \\
\text { Essay }\end{array}$ \\
\hline
\end{tabular}

This work is licensed under the Creative Commons Attribution-NonCommercial 4.0 International License. To view a copy of this license, visit http://creativecommons.org/licenses/by-nc/4.0/ or send a letter to Creative Commons, PO Box 1866, Mountain View, CA 94042, USA. 


\begin{tabular}{|c|c|c|}
\hline & $\begin{array}{l}\text { (We will be discussing this } \\
\text { article on Wed, so please } \\
\text { have it read by then...) } \\
\text { Watch: } \\
\underline{\text { Incorporating Sources into }} \\
\underline{\text { Your Essay | Library }}\end{array}$ & \\
\hline $\begin{array}{l}\text { Mon. 10/26: } \\
\text { Individual } \\
\text { meetings }\end{array}$ & & $\begin{array}{l}\text { - } \text { Peer Review } \\
\text { - } \text { Discussion Board }\end{array}$ \\
\hline $\begin{array}{l}\text { Mon. 11/2 } \\
\text { Wed. 11/4 } \\
\text { Synchronistic } \\
\text { Zoom Class }\end{array}$ & $\begin{array}{l}\text { Read: Bizup's "BEAM } \\
\text { Method of Research" (PDF is } \\
\text { on BB) } \\
\text { (We will be discussing this } \\
\text { reading Wednesday, so } \\
\text { please have it read by } \\
\text { then...) } \\
\text { Watch: } \\
\text { https://www.youtube.com/ } \\
\text { watch?v=CHgdvQYXQCk\&fe } \\
\text { ature=youtu.be }\end{array}$ & $\begin{array}{ll}\text { - } & \text { Blog Post } \\
\text { - } & \text { Peer Review } \\
\text { - } & \text { Discussion Board }\end{array}$ \\
\hline Mon. 11/9 & 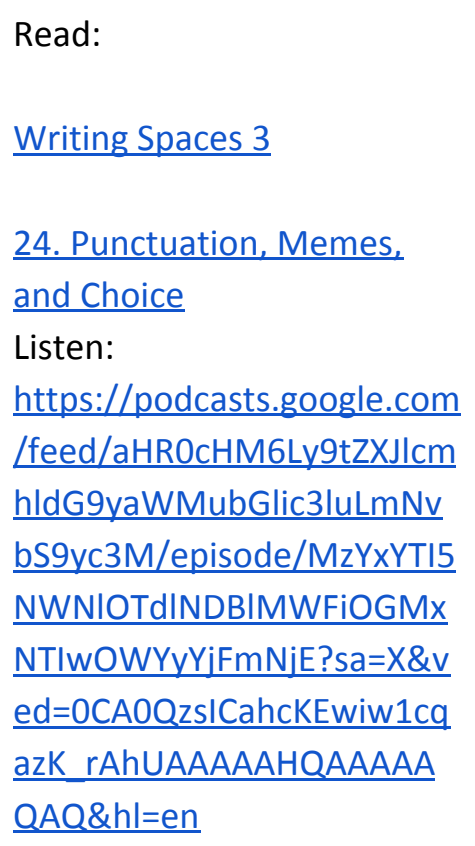 & $\begin{array}{ll}\text { - } & \text { Peer Review } \\
\text { - } & \text { Discussion Board }\end{array}$ \\
\hline
\end{tabular}

This work is licensed under the Creative Commons Attribution-NonCommercial 4.0 International License. To view a copy of this license, visit http://creativecommons.org/licenses/by-nc/4.0/ or send a letter to Creative Commons, PO Box 1866, Mountain View, CA 94042, USA. 


\begin{tabular}{|c|c|c|c|}
\hline $\begin{array}{l}\text { Mon. 11/16 } \\
\text { Wed. 11/18 } \\
\text { Synchronistic } \\
\text { Zoom Class }\end{array}$ & $\begin{array}{l}\text { Read: Flow, Coherence, } \\
\text { Unity } \\
\underline{\text { Transitional Language, }} \\
\underline{\text { Metalanguage, Seques }} \\
\underline{\text { Mark Danner Words in a }} \\
\underline{\text { Time of War (abridged) (We }} \\
\text { will be discussing Mark } \\
\text { Danner's piece during } \\
\text { Wednesday's class, so } \\
\text { please read it by then...) }\end{array}$ & $\begin{array}{ll}\text { - } & \text { Blog Post } \\
\text { - } & \text { Peer Review } \\
\text { - } & \text { Discussion Board } \\
\text { - } & \text { Sign up for individual } \\
& \text { meeting }\end{array}$ & \\
\hline $\begin{array}{l}\text { Mon. 11/23 } \\
\text { Individual } \\
\text { meetings }\end{array}$ & & - Peer Review & \\
\hline $\begin{array}{l}\text { Mon. 11/30 } \\
\text { Wed. 12/2 } \\
\text { Synchronistic } \\
\text { Zoom Class }\end{array}$ & $\begin{array}{l}\text { Read: Bizup's "The Ethics of } \\
\text { Style" (PDF posted on BB } \\
\text { and we will be discussing } \\
\text { reading so please have it } \\
\text { read by Wednesday...) }\end{array}$ & $\begin{array}{ll}\text { - } & \text { Blog Post } \\
\text { - } & \text { Peer Review } \\
\text { - } & \text { Discussion Board }\end{array}$ & $\begin{array}{l}\text { Due: Critical } \\
\text { Research } \\
\text { Analysis Sun. } \\
\text { 12/6 }\end{array}$ \\
\hline Mon. 12/7 & & $\begin{array}{l}\text { - Peer Review of another } \\
\text { student's CUNY Commons } \\
\text { Portfolio } \\
\text { - Discussion Board }\end{array}$ & \\
\hline
\end{tabular}

This work is licensed under the Creative Commons Attribution-NonCommercial 4.0 International License. To view a copy of this license, visit http://creativecommons.org/licenses/by-nc/4.0/ or send a letter to Creative Commons, PO Box 1866, Mountain View, CA 94042, USA. 